\title{
Risco climático da cana-de-açúcar cultivada na região Nordeste do Brasil
}

\author{
Vicente de P. R. da Silva ${ }^{1}$, Sonaly D. de Oliveira ${ }^{1}$, \\ Carlos A. C. dos Santos ${ }^{1} \&$ Madson T. Silva ${ }^{1}$
}

\begin{abstract}
RESUMO
Este estudo avalia os impactos das alterações climáticas sobre o zoneamento agrícola de risco climático da cultura da cana-de-açúcar cultivada na região Nordeste do Brasil, baseado nos relatórios do IPCC. Combinado com técnicas de geoprocessamento (SIG) o modelo de balanço hídrico foi utilizado para identificar as áreas da região de estudo nas quais a cultura poderá sofrer restrições de rendimento devido às mudanças climáticas. Os dados utilizados no estudo foram as séries históricas de precipitação com no mínimo 30 anos de dados diários, coeficientes de cultura, evapotranspiração potencial e duração do ciclo da cultura. Adotou-se, como critério de corte para o Índice de Satisfação das Necessidades de Água para a cultura (ISNA) definido como a relação entre a evapotranspiração real e a evapotranspiração máxima (ETr/ETm) o valor 0,65. Os cenários utilizados nas simulações sem aumento na temperatura do ar e com aumento de 1,5; 3 e $5^{\circ} \mathrm{C}$ foram associados às alterações na precipitação de $\pm 10, \pm 25$ e $\pm 40 \%$. Os resultados indicam que existe uma diferença relevante entre os cenários de aquecimento e as condições climáticas atuais, em termos dos efeitos projetados da variação de temperatura sobre as áreas cultivadas com a cana-de-açúcar na região de estudo.
\end{abstract}

Palavras-chave: mudanças climáticas, zoneamento agrícola, evapotranspiração, precipitação

\section{Climate risk of sugarcane grown in the northeast region of Brazil}

\begin{abstract}
This study evaluates the impacts of climate change on the agricultural zoning of climatic risk of sugarcane crop grown in Northeastern Brazil based on IPCC reports. The water balance model combined with geospatial technologies (GIS) was used to identify regional areas where the crop will suffer yield reduction due to climate changes. The data used in the study were time series of precipitation at least 30 years of daily data, crop coefficients, potential evapotranspiration and duration of the crop cycle. A limit value of 0.65 was adopted for the water requirement satisfaction index (WRSI), which is defined as the ratio between actual evapotranspiration and maximum evapotranspiration (ETr/ETm). The scenarios used in simulations without an increase in air temperature and an increase of $1.5,3$ and $5{ }^{\circ} \mathrm{C}$ were associated with changes in precipitation of $\pm 10, \pm 25$ and $\pm 40 \%$. The results indicate that there is significant difference between the warming scenarios and the current weather conditions, in terms of the projected effects of temperature variation on the areas cultivated with sugarcane in the study region.
\end{abstract}

Key words: climate change, agricultural zoning, evapotranspiration, rainfall 


\section{INTRODUÇÃO}

$\mathrm{O}$ aumento nas emissões dos gases de efeito estufa em decorrência da ação antrópica, tem-se intensificado e pode trazer consequências negativas para os ecossistemas naturais. Por este motivo, as mudanças climáticas e o aquecimento global começam a ser amplamente estudados por vários pesquisadores em diferentes partes do mundo (Lohmann et al., 2006; Chavas et al., 2009; Harmsen et al., 2009, Campos et al., 2010). O Intergovernmental Panel on Climate Change (IPCC) indicou, por meio de modelos matemáticos baseados em dados registrados nos oceanos, biosfera e atmosfera, um aumento entre 1,4 e $5,8^{\circ} \mathrm{C}$ da temperatura global até o final do século XXI (IPCC, 2001 e 2007). Para a América Latina está previsto um aumento de 2 a $6{ }^{\circ} \mathrm{C}$ na temperatura da superfície terrestre e aumento da frequência e intensidade de eventos extremos, tais como: ondas de calor/frio, secas, enchentes e, sobretudo, furacões e tempestades tropicais. A magnitude dessas previsões ainda é incerta, pois pouco se sabe sobre os processos de troca de calor, de carbono e de radiação solar entre os diversos ecossistemas (Silva et al., 2010).

No setor econômico a pesca, a agricultura e o turismo tendem a ser os mais afetados em virtude dos desastres naturais. Especificamente no Brasil, espera-se elevação de aproximadamente $4{ }^{\circ} \mathrm{C}$ na temperatura da superfície terrestre; aumento de 10 a $15 \%$ na precipitação durante o outono e redução durante o verão, além da intensificação de eventos como El Niño e La Niña na região Sul do País (IPCC, 2007). O clima é o fator que oferece os maiores desafios para o manejo correto das culturas. Para que haja crescimento e desenvolvimento das plantas é necessário que os aspectos do clima, tais como temperatura média do ar (diurna e noturna), precipitação pluvial e radiação solar, estejam de acordo com as exigências da cultura. $\mathrm{O}$ manejo racional do solo e a água através de técnicas convencionais são imprescindíveis para a sustentabilidade, de forma a manter esses recursos com qualidade e quantidade suficientes para obter níveis satisfatórios de produtividade. Os cenários climáticos traçados pelo IPCC, tais como as secas, inundações, tempestades, doenças, extinção de espécies, aumento do nível do mar, são preocupantes para a agricultura, a biodiversidade e os recursos hídricos do planeta. Tal cenário é ainda mais crítico para os países pobres, justamente os menores emissores de gases do efeito estufa devido, fundamentalmente, às suas posições geográficas (Campos et al., 2010). As mudanças climáticas no planeta envolvem fatores internos e externos ao sistema terra-atmosfera que podem afetar a produtividade das culturas, em todas as partes do mundo.

No Brasil a cana-de-açúcar, além de outras culturas, pode sofrer drásticas reduções em face do aquecimento global. A cultura da cana teve sua expansão no Nordeste Brasileiro (NEB) no período de 1970 a 1975, com recursos do programa do álcool (Proálcool) principalmente nos tabuleiros costeiros por se tratar de uma região mais favorecida por chuvas em determinados períodos do ano. Entretanto, a má distribuição e a redução dos volumes de chuvas têm sido uma constante na região, nos últimos anos, trazendo prejuízos à cultura, com a mortalidade de soqueiras, forçando uma renovação precoce do canavial (Farias et al., 2008). O Brasil é um dos maiores produtores de cana-de-açúcar no mundo, com 8,36 milhões de hectares de área cultivada e uma produção de 5,58 milhões toneladas de colmos, 26,6 milhões de metros cúbicos de álcool e 30,0 milhões de toneladas de açúcar (EPE, 2008).

As regiões tradicionalmente canavieiras, como as regiões dos Tabuleiros Costeiros e Zona da Mata do Nordeste do Brasil, não dispõem mais de área para expansão tornando-se imprescindível o manejo adequado desta cultura visando ao aumento de sua produtividade (Almeida et al., 2008). Isto implica em conhecer os padrões de crescimento de cada variedade da cultura fazendo com que as fases de máximo desenvolvimento coincidam com os períodos de maior disponibilidade hídrica e radiação sola, permitindo que ela expresse todo o seu potencial genético. No Brasil, poucos estudos sobre a fisiologia da cana-de-açúcar têm sido conduzidos com a finalidade de se verificar a resposta dessa cultura às mudanças climáticas. A maioria dessas informações é obtida por meio de trabalhos realizados em outros países. Neste sentido, o presente trabalho tem o objetivo de avalia os impactos das alterações climáticas de temperatura do ar e precipitação sobre o zoneamento agrícola de risco climático da cultura da cana-de-açúcar cultivada nas condições edafoclimátidas da região Nordeste do Brasil.

\section{Material e Métodos}

O risco climático do cultivo da cana-de-açúcar (Saccharum officinarum L.) foi realizado para toda a região Nordeste do Brasil. A área do NEB é de aproximadamente $1.558196 \mathrm{~km}^{2}$, equivalente a $18 \%$ do território nacional e é a região que possui a maior costa litorânea. A precipitação média anual na região varia entre $400 \mathrm{~mm}$ na parte semiárida a $1500 \mathrm{~mm}$ no litoral, com seis meses secos; o clima é quente e úmido, com chuvas de outono a inverno, com seca atenuada (Silva, 2004).

Neste estudo utilizou-se o modelo SARRA (Systeme d'Analyse Regionale dês Risques Agroclimatiques) para avaliar os riscos de seca e seus impactos na agricultura na área de estudo. Como modelo determinístico ele é bastante utilizado para estabelecer o zoneamento e a análise de risco de cereais (Baron et al., 2005) e pode ser utilizado em escalas de tempo diárias (Kouressy et al., 2008) na análise de impactos climáticos sobre o crescimento e o rendimento de cereais em ambientes tropicais semiáridos (Sultan et al., 2005; Baron et al., 2005). Este modelo contém um módulo que executa o balanço hídrico no solo, usado para se obter as necessidades hídricas e o Índice de Satisfação da Necessidade de Água para a cultura (ISNA) definido como a relação entre a evapotranspiração real (ETr) e evapotranspiração máxima $(\mathrm{ETm}),(\mathrm{ISNA}=\mathrm{ETr} / \mathrm{ETm})$. Este índice foi desenvolvido, originalmente, pela Organização para a Alimentação e Agricultura (FAO) a fim de avaliar o rendimento das culturas com limitações hídricas durante o período de crescimento (Doorenbos \& Pruitt, 1977).

O modelo SARRA, tal como o DSSAT (Sistema de Suporte à Decisão para Transferência de Agrotecnologia) tem sido utilizado intensivamente em muitas partes do mundo visando avaliar o rendimento das culturas em ambientes semiáridos (Sultan et al., 2005; Mishra et al., 2008; Thornton et al., 2009). O modelo SARRA foi utilizado neste estudo com o propósito 
de avaliar os riscos climáticos da cana-de-açúcar cultivada em sistema de sequeiro no NEB. Adicionalmente, também foram utilizados, nesta pesquisa, dois softwares computacionais: (i) Estima-T: estima a temperatura do ar (Cavalcanti et al., 2006) e (ii) Sevap: estima a evapotranspiração potencial por diversos métodos (Silva et al., 2005).

Neste estudo foram investigados os cenários de mudanças climáticas globais para os aumentos de temperatura de 1,5; 3,0 e $5,0^{\circ} \mathrm{C}$. Essas variações de temperatura do ar foram associadas às ocorrências dos fenômenos El Niño e La Niña, com alterações de acréscimo e decréscimo de 10, 25 e 40\% na precipitação. Na aplicação do modelo não foi considerada qualquer evolução tecnológica, seja no manejo das culturas ou no seu melhoramento genético nem qualquer adaptação fisiológica das plantas às novas condições. Os cenários agrícolas foram simulados utilizando-se o Sistema de Análise Regional dos Riscos Agroclimáticos, com base no software SARRA (Baron et al., 1996). Tal modelo estima componentes do balanço hídrico de uma superfície vegetada em solo estratificado através dos dados diários de precipitação pluvial e evapotranspiração potencial, além de coeficientes de cultivo $(\mathrm{Kc})$ que descrevem o crescimento de culturas e as características de solo. Neste modelo a infiltração e a redistribuição de água no solo são governadas pela equação de Darcy-Buckingham e a evapotranspiração real é dada em função da evapotranspiração máxima e da umidade do solo. A espacialização das estações meteorológicas utilizadas no estudo é exibida na Figura 1.

Os valores de ISNA foram estimados para o período fenológico compreendido entre o perfilhamento e o alongamento dos colmos (período mais crítico ao déficit hídrico) com

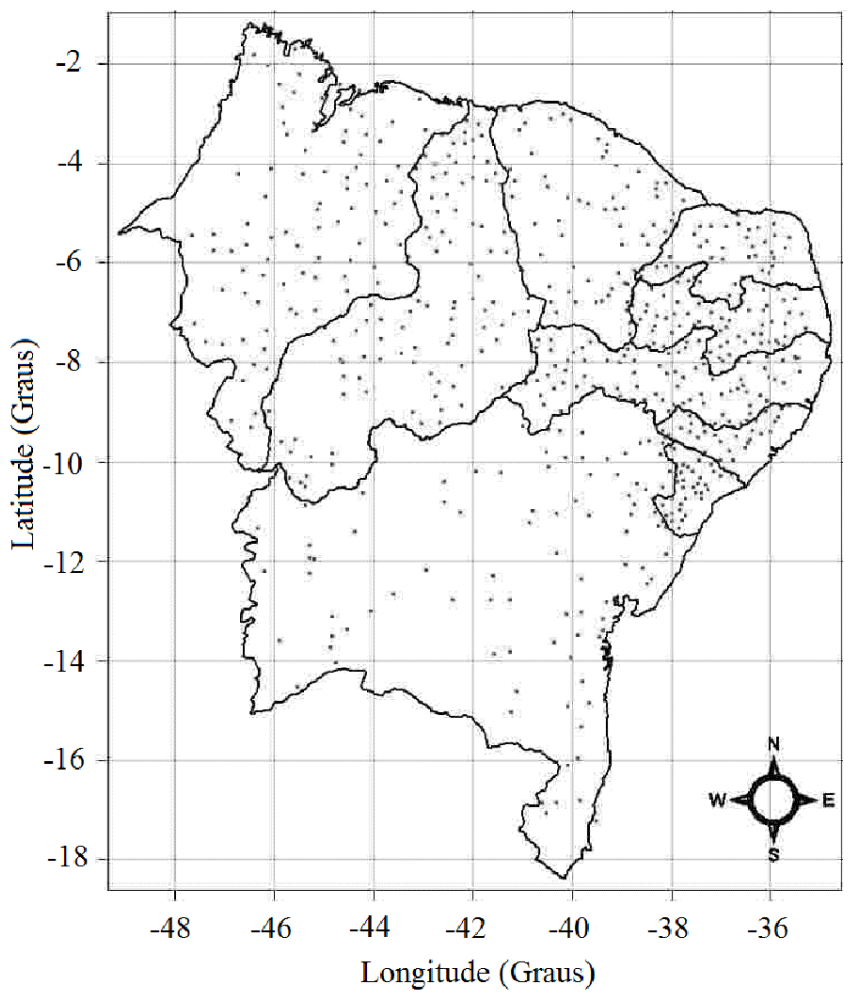

Figura 1. Espacialização das estações meteorológicas utilizadas no estudo frequência mínima de $80 \%$ nos anos utilizados em cada estação pluviométrica. Cada valor de ISNA observado durante esta fase foi associado à localização geográfica da respectiva estação para posterior espacialização e, consequentemente, foi computado o valor das áreas, de acordo com os critérios de risco climático associados ao cultivo da cana-de-açúcar. A partir desses valores estabeleceu-se a função de frequência do ISNA e se selecionou a data em que o valor calculado é maior ou igual ao critério de risco adotado (ISNA $\geq 0,65$ ) em $80 \%$ dos casos. Para a caracterização do risco climático obtido ao longo dos períodos de simulações foram estabelecidas três classe de ISNA conforme (Amaral \& Silva, 2007): (i) ISNA $\geq 0,65$ - a cultura da cana-de-açúcar de sequeiro está exposta a um baixo risco climático; (ii) 0,55 $\leq$ ISNA $<0,65$ - a cana-de-açúcar de sequeiro está exposta a médio risco climático e (iii) ISNA < 0,55 - a cultura da cana-de-açúcar de sequeiro está exposta a um alto risco climático.

\section{Resultados E Discussão}

A distribuição espacial do ISNA nas condições climáticas atuais para todos os meses do ano é exibida na Figura 2, enquanto os efeitos dos cenários climáticos de temperatura e precipitação sob o cultivo da cana-de-açúcar no NEB são apresentados em tabelas. É importante observar que esta análise não leva em consideração o rendimento da cultura em cada cenário climático; contudo, avalia os impactos das mudanças climáticas na área favorável ao cultivo da cana-de-açúcar na área de estudo.

As análises apresentadas a seguir estão focadas para o segundo decêndio de cada mês do ano, haja vista que a cultura da cana-de-açúcar é de ciclo longo e sua aptidão climática depende da região, face às condições edafoclimáticas de cada localidade.

A distribuição espacial do risco climático da cana-de-açúcar cultivada no segundo decêndio de todos os meses do ano nas condições climáticas atuais de temperatura e precipitação evidencia uma faixa no litoral do NEB com baixo risco climático, mesclada com alguns núcleos de médio risco nos meses de janeiro a março (Figura 2A). No mês de janeiro essa faixa se estende desde a costa do estado do Rio Grande do Norte até o sul da Bahia, com um núcleo de médio risco climático localizado ao Norte do estado do Maranhão. No mês de março aparece apenas uma pequena faixa com médio risco climático (Figura 2C), bem menor do que aquela do mês de fevereiro (Figura 2B); entretanto, nos meses de abril (Figura 2D) e maio (Figura 2E) praticamente todo o NEB apresenta alto risco climático, exceto para uma pequena faixa com médio risco climático na costa do estado da Bahia.

A partir do mês de junho (Figura $2 \mathrm{~F}$ ) começam a aparecer áreas com médio risco climático ao cultivo da cana-de-açúcar na costa oeste da região, que se expande com risco climático médio e baixo nos meses julho e agosto atingindo, inclusive, a parte norte do NEB neste último mês (Figura 2H). Essa extensa área com baixo e médio risco climático localizada no mês de agosto começa a decrescer nos meses subsequentes atingindo a menor porção no mês de dezembro, localizada ao norte do estado do Maranhão (Figura 2L). O decréscimo desses riscos 
A.

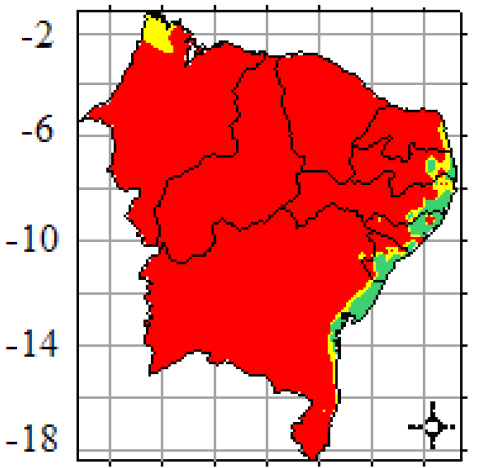

E

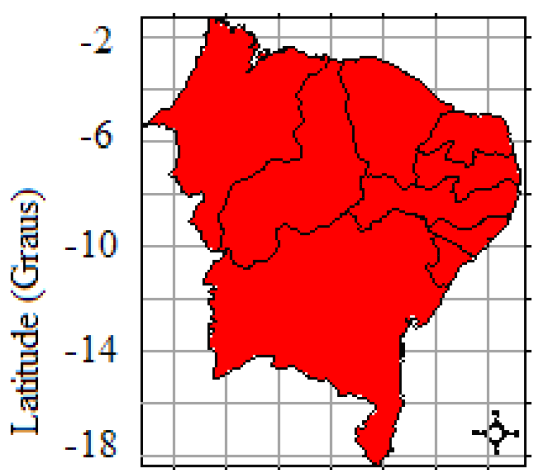

I.

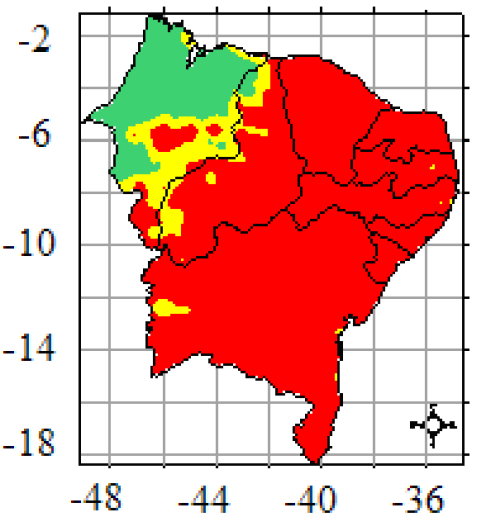

B.

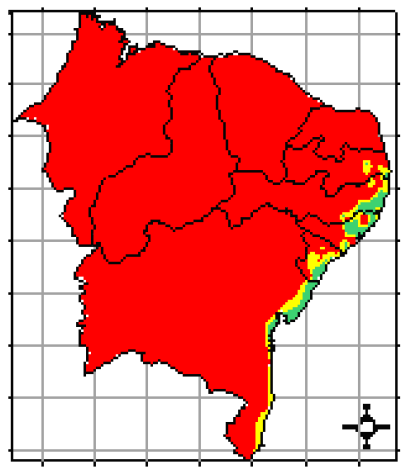

F.

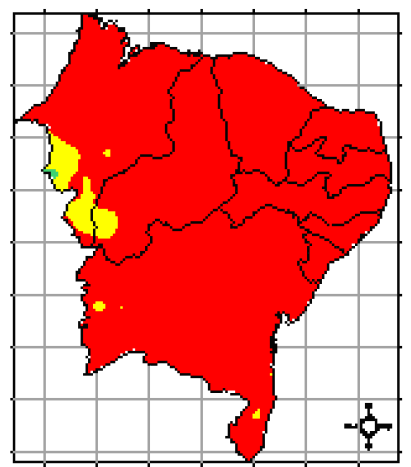

$\mathrm{J}$.

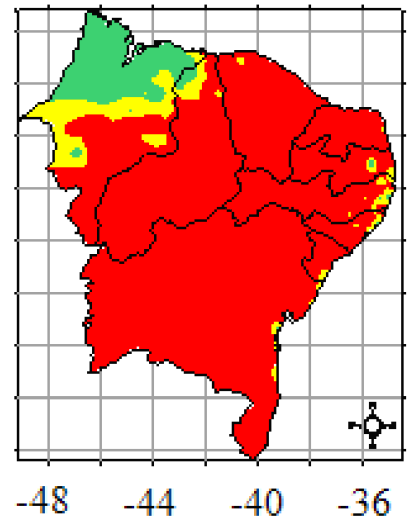

C.

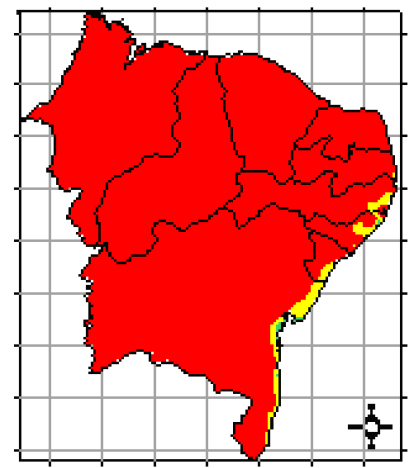

G.

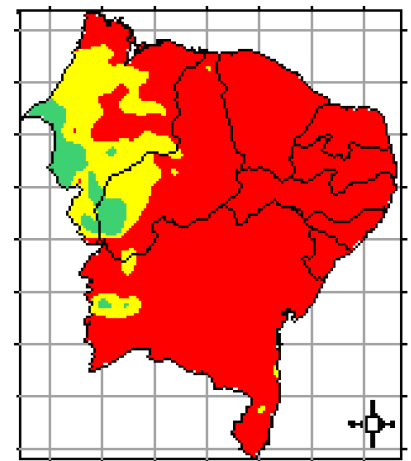

$\mathrm{K}$.

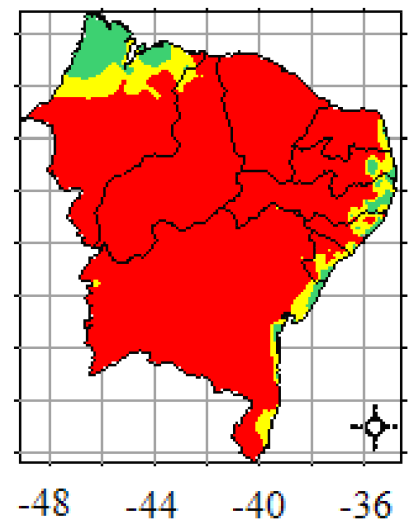

D.

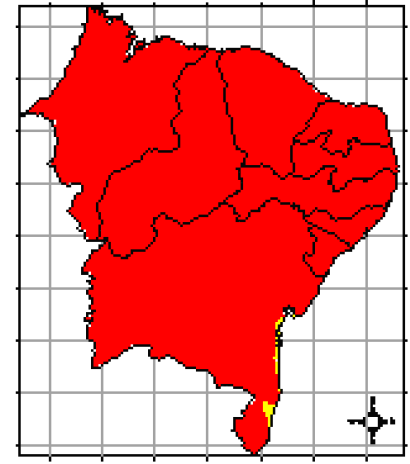

$\mathrm{H}$.

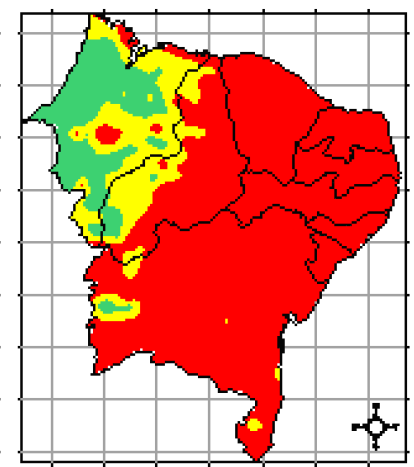

L.

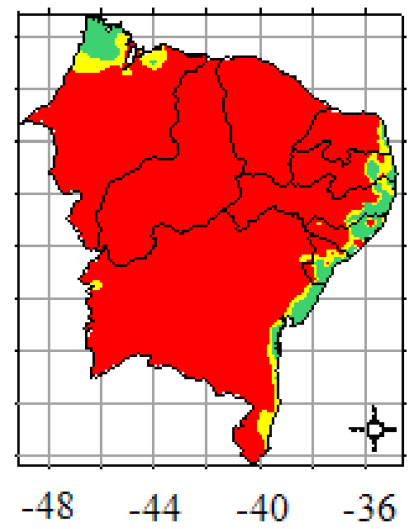

Longitude (Graus)

\section{Alto risco}

\section{Médio risco}

Baixo risco

Figura 2. Risco climático da cana-de-açúcar cultivada no segundo decêndio dos meses janeiro (A), fevereiro (B), março $(C)$, abril $(D)$, maio $(E)$, junho $(F)$, julho $(G)$, agosto $(H)$, setembro $(\mathrm{I})$, outubro $(\mathrm{J})$, novembro $(\mathrm{K})$ e dezembro (L) na região Nordeste do Brasil, para as condições climáticas atuais

climáticos é acompanhado do aumento das áreas favoráveis ao cultivo da cana-de-açúcar a partir do mês de outubro (Figura 2J) na costa leste do NEB, até atingir a maior porção no mês de dezembro. Resultados semelhantes foram obtidos por Silva et al. (2010) quando analisaram os impactos do aquecimento global sobre a cultura do feijão na região Nordeste do Brasil.

A Tabela 1 exibe a área agrícola da cana-de-açúcar cultivada na região Nordeste do Brasil com alto risco (AR), médio risco (MR) e baixo risco climático (BR) para o cultivo da cana-deaçúcar cultivada na região Nordeste do Brasil para as condições climáticas atuais (CCA). Observa-se que as áreas com baixo e médio riscos climáticos são inferiores quando comparadas àquelas com alto risco climático para cultivo da cana-de-açúcar no NEB. A maior área apta ao cultivo da cana-de-açúcar ocorre nos decêndios de agosto (com médio risco climático de 14,71\% e baixo risco de $11,73 \%$ ) e setembro (com médio risco climático de $10,60 \%$ e baixo risco de $11,19 \%$ ). Por outro lado, o segundo decêndio de julho apresenta uma área bastante elevada com médio risco climático, de $206.781,33 \mathrm{~km}^{2}$, que corresponde a $13,52 \%$ da área total do NEB.

As áreas com baixo risco climático neste decêndio são relativamente menores, com 3,76\% da área do NEB. Por outro 
Tabela 1. Área agrícola (\%) da cana-de-açúcar cultivada na região Nordeste do Brasil com alto risco $(A R)$, médio risco $(M R)$ e baixo risco climático $(B R)$ nas condições climáticas atuais (CCA)

\begin{tabular}{cccc}
\hline Mês de semeadura & AR & MR & BR \\
Janeiro & 94,05 & 2,79 & 3,16 \\
Fevereiro & 95,61 & 2,53 & 1,86 \\
Março & 97,55 & 2,07 & 0,38 \\
Abril & 99,21 & 0,78 & 0,01 \\
Maio & 99,85 & 0,15 & 0,00 \\
Junho & 96,74 & 3,22 & 0,05 \\
Julho & 82,72 & 13,52 & 3,76 \\
Agosto & 73,55 & 14,71 & 11,73 \\
Setembro & 78,21 & 10,60 & 11,19 \\
Outubro & 86,25 & 5,72 & 8,03 \\
Novembro & 87,87 & 7,98 & 4,15 \\
Dezembro & 90,90 & 4,46 & 4,64 \\
\hline
\end{tabular}

lado, o segundo decêndio de maio apresentou a menor área apta para o cultivo da cana-de-açúcar, com baixo e médio riscos climáticos de 0 e $0,15 \%$, respectivamente.

A Tabela 2 apresenta a variação percentual das áreas agrícolas da cana-de-açúcar cultivada na região Nordeste do Brasil com alto risco (AR), médio risco (MR) e baixo risco climático (BR) com redução de $10 \%$ na precipitação pluvial, sem aumento na temperatura do ar e aumentos de $1,5,3$ e $5^{\circ} \mathrm{C}$, condições em que se verifica que as alterações nas mudanças climáticas podem ser ainda mais críticas para as áreas com baixo risco climático ao longo do período do cultivo da cana-deaçúcar no NEB. No cenário de redução da precipitação pluvial sem aumento na temperatura em todos os meses, as áreas com médio e baixo risco climático se apresentaram superiores as dos demais cenários, com aumentos na temperatura.

Agosto é o mês mais favorável com área apta ao cultivo da cana-de-açúcar de $115.027,57 \mathrm{~km}^{2}$ correspondendo a 7,5\% da área do NEB e aproximadamente $250.000 \mathrm{~km}^{2}$ de área com médio risco climático, que equivale a $16,3 \%$ da área total do NEB. A área apta é ainda maior para as condições climáticas atuais pois possui $225.058,93 \mathrm{~km}^{2}$ que correspondem a $14,71 \%$ da área total da região de estudo. Os segundos decêndios do período abril - junho não possuem área alguma com baixo risco climático; apenas os meses de abril e junho exibem pequenas áreas com médio risco climático de 0,4 e $2 \%$, respectivamente; o mês de maio é, para esse cenário, o mais crítico, apresentando $100 \%$ de área inapta ao cultivo da cana- de-açúcar, correspondendo a 1.558,196 km². Para os cenários de aumento na temperatura do ar de $1,5^{\circ} \mathrm{C}$ os segundos decêndios dos meses de abril e maio exibem $100 \%$ de áreas com alto risco climático ao cultivo da cana-de-açúcar; entretanto, a maior área com médio risco climático ocorre no mês de agosto com $242.207,14 \mathrm{~km}^{2}$ equivalente a $15,8 \%$ da área total do NEB. Com base nesta condição de menor oferta hídrica para o mesmo cenário de aumento de $1,5^{\circ} \mathrm{C}$, as áreas com baixo risco climático sofrem uma variação de 0 a 5,7\% da área total, tendo o mês de setembro a maior área apta com $86.849,41 \mathrm{~km}^{2}$.

Aumentando a temperatura do ar para $3{ }^{\circ} \mathrm{C}$ a área com baixo risco climático tem uma variação de 0 a $2,5 \%$, com o mês de outubro exibindo uma área apta de $37.483,12 \mathrm{~km}^{2}$; para este mesmo cenário o segundo decêndio do mês de agosto tem as áreas com médio risco climático reduzidas para $182.905,52 \mathrm{~km}^{2}$. Estudos realizados por Oliveira et al. (2012) sobre os impactos das alterações climáticas na cana-de-açúcar cultivada em sistema de sequeiro na região Nordeste do Brasil indicaram que o aquecimento global associado às anomalias de precipitação pluvial pode trazer graves consequências para a economia da região, face à inaptidão dessa cultura para uma produção economicamente viável sob níveis elevados de temperatura do ar.

Nos cenários com um aumento na temperatura de $5{ }^{\circ} \mathrm{C}$ para o mesmo período simulado, as áreas com médio risco climático reduzem ainda mais apresentando $105.374,18 \mathrm{~km}^{2}$ e o mês de setembro passa a apresentar a maior área com médio risco com $7,7 \%$, que corresponde a $117.655,2 \mathrm{~km}^{2}$ da área total do NEB. Neste mesmo cenário verifica-se claramente uma enorme redução de áreas com baixo risco climático enquanto o segundo decêndio do mês de outubro é o menos pessimista, com 1,7\% da área do NEB, os meses de março a maio apresentam áreas mais reduzidas, como consequência dos cenários de aumento na temperatura do ar. Segundo estudos realizados por Silva (2010) quanto maior a anomalia menos apta se tornará a região, até o limite máximo de tolerância biológica ao calor ou à seca provocada pelo aumento térmico. De modo geral, o cenário de redução na precipitação sem aumento da temperatura é o que apresenta os melhores resultados para as áreas favoráveis ao cultivo da cana-de-açúcar quando comparado com os dados apresentados na Tabela 1 (condições climáticas atuais) enquanto os demais cenários com aumento na temperatura afetam, de

Tabela 2. Área agrícola (\%) da cana-de-açúcar cultivada na região Nordeste do Brasil com alto risco (AR), médio risco $(M R)$ e baixo risco climático $(B R)$ com redução de $10 \%$ na precipitação pluvial e sem aumento na temperatura do ar e aumentos de 1,$5 ; 3$ e $5{ }^{\circ} \mathrm{C}$

\begin{tabular}{|c|c|c|c|c|c|c|c|c|c|c|c|c|}
\hline \multirow{2}{*}{$\begin{array}{l}\text { Mês de } \\
\text { semeadura }\end{array}$} & AR & MR & BR & AR & MR & BR & $\overline{A R}$ & MR & BR & $\overline{A R}$ & MR & BR \\
\hline & \multicolumn{3}{|c|}{ Sem aumento } & \multicolumn{3}{|c|}{ Aumento de $1,5^{\circ} \mathrm{C}$} & \multicolumn{3}{|c|}{ Aumento de $3^{\circ} \mathrm{C}$} & \multicolumn{3}{|c|}{ Aumento de $5^{\circ} \mathrm{C}$} \\
\hline Janeiro & 94,8 & 2,6 & 2,5 & 97,7 & 1,3 & 1,0 & 97,9 & 1,7 & 0,4 & 99,3 & 0,7 & 0,1 \\
\hline Fevereiro & 96,6 & 2,3 & 1,1 & 98,7 & 1,0 & 0,2 & 99,0 & 1,0 & 0,1 & 99,8 & 0,2 & 0,0 \\
\hline Março & 98,4 & 1,4 & 0,2 & 99,9 & 0,1 & 0,0 & 99,9 & 0,1 & 0,0 & 100,0 & 0,0 & 0,0 \\
\hline Abril & 99,6 & 0,4 & 0,0 & 100,0 & 0,0 & 0,0 & 100,0 & 0,0 & 0,0 & 100,0 & 0,0 & 0,0 \\
\hline Maio & 100,0 & 0,0 & 0,0 & 100,0 & 0,0 & 0,0 & 100,0 & 0,0 & 0,0 & 100,0 & 0,0 & 0,0 \\
\hline Junho & 98,0 & 2,0 & 0,0 & 99,6 & 0,4 & 0,0 & 99,8 & 0,2 & 0,0 & 99,9 & 0,1 & 0,0 \\
\hline Julho & 85,7 & 11,9 & 2,5 & 93,8 & 5,6 & 0,6 & 95,6 & 4,1 & 0,3 & 97,6 & 2,3 & 0,1 \\
\hline Agosto & 76,1 & 16,3 & 7,5 & 82,5 & 15,8 & 1,7 & 87,7 & 12,0 & 0,3 & 93,1 & 6,9 & 0,1 \\
\hline Setembro & 80,8 & 10,0 & 9,2 & 86,8 & 7,5 & 5,7 & 89,5 & 8,1 & 2,4 & 91,4 & 7,7 & 0,9 \\
\hline Outubro & 87,8 & 5,0 & 7,1 & 90,4 & 5,6 & 4,0 & 92,5 & 5,0 & 2,5 & 94,1 & 4,2 & 1,7 \\
\hline Novembro & 89,6 & 7,1 & 3,3 & 94,0 & 4,1 & 1,9 & 95,9 & 3,0 & 1,2 & 97,0 & 2,1 & 0,9 \\
\hline Dezembro & 92,2 & 4,1 & 3,7 & 95,8 & 2,7 & 1,5 & 96,5 & 2,9 & 0,6 & 98,1 & 1,8 & 0,1 \\
\hline
\end{tabular}


forma mais acentuada, as áreas agricultáveis, tendo em vista a menor oferta hídrica provocada, por exemplo, pelo fenômeno El Niño.

Analisando os resultados apresentados na Tabela 3 observase, para os cenários sem aumento na temperatura, que as áreas com baixo e médio riscos climáticos apresentam uma manutenção ou crescimento das áreas quando comparados aos resultados obtidos nas CCA. Os meses de agosto e setembro são os que apresentaram as condições mais favoráveis ao cultivo da cana-de-açúcar exibindo, no segundo decêndio do mês de agosto, um acréscimo das áreas com baixo risco climático de 11,73 para $14,7 \%$, que corresponde a um aumento de $179.482,73$ para $224.621,80 \mathrm{~km}^{2}$ enquanto o mês de setembro apresenta uma área com baixo risco de $12,5 \%$ que equivale a 191.627,76 $\mathrm{km}^{2}$ contra $10,6 \%$ das CCA.

Para as áreas com médio risco climático a diferença entre os cenários sem aumento na temperatura e as CCA no mês de agosto, é de apenas $0,7 \%$ e para o mês de setembro é de $0,9 \%$, o que resulta praticamente na manutenção dessas áreas. Apesar de uma oferta hídrica maior, o segundo decêndio dos meses de abril e maio anula as áreas consideradas favoráveis ao cultivo da cana-de-açúcar apresentando apenas uma pequena área com médio risco de 1,4 e $0,6 \%$, respectivamente. É evidente que o mês de maio é o que apresenta a maior área inapta ao cultivo com $1.520 .003,82 \mathrm{~km}^{2}$, equivalente a $99,4 \%$ de toda a área do NEB.

Analisando o cenário com aumento na temperatura do ar de $1,5^{\circ} \mathrm{C}$ verifica-se, para o período simulado no mês de agosto, que as áreas com médio risco climático aumentam em relação ao cenário sem aumento na temperatura, aumentando seu percentual para $17,3 \%$. O segundo decêndio do mês de setembro, persistindo o aumento na temperatura de $1,5^{\circ} \mathrm{C}$ apresenta a maior área apta ao cultivo da cana-de-açúcar com $8 \%$ da área do NEB correspondendo a 122.019,51 $\mathrm{km}^{2}$. Por outro lado e mesmo diante de um acréscimo na precipitação pluvial, as áreas com alto risco climático para o mês de maio afetam todo o NEB. Com um aumento na temperatura do ar de $3{ }^{\circ} \mathrm{C}$ o mês de maio possui o mesmo comportamento; entretanto, para os quatro períodos simulados do mês de março ao mês de junho, as áreas com baixo risco climático continuam nulas porém as áreas com médio risco apresentam uma pequena variação de 0,1 a $1 \%$ da área total do NEB; apesar disto, o segundo decêndio do mês de agosto apresenta a maior área favorável ao cultivo da cana-de-açúcar (com médio risco climático de $16,6 \%$ e baixo risco de $2,1 \%$ ).

As áreas com médio risco climático para este mesmo período sofrem uma pequena redução quando comparadas com as do cenário de aumento na temperatura de $5{ }^{\circ} \mathrm{C}$ de 16,6 para $14,3 \%$, representando uma diferença de $2,3 \%$ que equivale a $35.246,13 \mathrm{~km}^{2}$. Apesar da maior oferta hídrica, este aumento na temperatura exibe os efeitos negativos das mudanças climáticas sobre as áreas consideradas aptas ao cultivo da cana-de-açúcar na região nordeste do Brasil. Analisando os impactos das alterações climáticas sobre a área de cultivo e a produtividade de milho e feijão no Nordeste do Brasil usando modelagem agrometeorológica, Campos et al. (2010) observaram que o aumento na temperatura do ar pode afetar significativamente a disponibilidade de área agricultável das culturas de feijão no estado da Paraíba; já para os cenários sem aumento na temperatura o segundo decêndio do mês de agosto continua sendo o mais favorável para o cultivo da cana-de-açúcar na região Nordeste do Brasil, exibindo áreas com baixo risco climático de $2,3 \%$ e médio risco de $16,2 \%$ (Tabela 4 ); contudo, o período simulado para o mês de setembro apresenta a maior área apta ao cultivo da cultura com $98.306,95 \mathrm{~km}^{2}$, que equivale a $6,4 \%$ da área total do NEB.

Verifica-se uma redução significativa nas áreas de baixo e médio risco climático e, consequentemente, um aumento nas áreas de alto risco para os meses compreendidos entre março e junho, exibindo áreas aptas nulas e uma variação de 0,1 a $0,6 \%$ das áreas com médio risco climático. $\mathrm{O}$ segundo decêndio do mês de maio permanece sendo o mais crítico, apresentando $100 \%$ de áreas inaptas ao cultivo da cana-de-açúcar na região. Analisando o cenário de aumento na temperatura de $1,5^{\circ} \mathrm{C}$, o período de março a junho continua apresentando áreas com baixo risco climático nulo; no entanto, as áreas inaptas ao cultivo da cultura chegam a $100 \%$ durante o período simulado nos meses de abril a junho. As áreas agricultáveis favoráveis ao cultivo compreendem os meses de agosto (com médio risco climático de $9,6 \%$ e baixo risco de $0,1 \%$ ), setembro (com médio risco climático de $7,5 \%$ e baixo risco de $2,3 \%$ ) e outubro (com médio risco climático de $5,1 \%$ e baixo risco de $2,5 \%$ ). Analisando o cultivo do algodão herbáceo no sistema de sequeiro no Nordeste do Brasil no cenário de mudanças climáticas, Silva et al. (2012) verificaram que no cenário de aquecimento de $1,5^{\circ} \mathrm{C}$ na temperatura média do ar a disponibilidade de áreas favoráveis

Tabela 3. Área agrícola (\%) da cana-de-açúcar cultivada na região Nordeste do Brasil com alto risco (AR), médio risco (MR) e baixo risco climático (BR) com aumento de $10 \%$ na precipitação pluvial e sem aumento na temperatura do ar e aumentos de $1,5,3$ e $5{ }^{\circ} \mathrm{C}$

\begin{tabular}{|c|c|c|c|c|c|c|c|c|c|c|c|c|}
\hline \multirow{2}{*}{$\begin{array}{c}\text { Mês de } \\
\text { semeadura }\end{array}$} & $\mathbf{A R}$ & MR & BR & AR & MR & BR & AR & MR & BR & AR & MR & BR \\
\hline & \multicolumn{3}{|c|}{ Sem aumento } & \multicolumn{3}{|c|}{ Aumento de $1,5^{\circ} \mathrm{C}$} & \multicolumn{3}{|c|}{ Aumento de $3^{\circ} \mathrm{C}$} & \multicolumn{3}{|c|}{ Aumento de $5^{\circ} \mathrm{C}$} \\
\hline Janeiro & 92,7 & 3,5 & 3,8 & 95,1 & 2,4 & 2,5 & 96,7 & 2,0 & 1,2 & 98,3 & 1,5 & 0,2 \\
\hline Fevereiro & 94,7 & 3,1 & 2,1 & 96,6 & 2,4 & 1,0 & 98,1 & 1,7 & 0,2 & 99,4 & 0,6 & 0,0 \\
\hline Março & 96,8 & 2,6 & 0,5 & 98,4 & 1,4 & 0,1 & 99,5 & 0,5 & 0,0 & 99,9 & 0,1 & 0,0 \\
\hline Abril & 98,5 & 1,4 & 0,0 & 99,6 & 0,4 & 0,0 & 99,9 & 0,1 & 0,0 & 100,0 & 0,0 & 0,0 \\
\hline Maio & 99,4 & 0,6 & 0,0 & 100,0 & 0,0 & 0,0 & 100,0 & 0,0 & 0,0 & 100,0 & 0,0 & 0,0 \\
\hline Junho & 95,7 & 4,2 & 0,1 & 97,7 & 2,3 & 0,0 & 99,0 & 1,0 & 0,0 & 99,5 & 0,5 & 0,0 \\
\hline Julho & 79,9 & 15,1 & 5,1 & 85,6 & 12,0 & 2,4 & 92,1 & 7,0 & 0,9 & 94,9 & 4,6 & 0,4 \\
\hline Agosto & 71,4 & 14,0 & 14,7 & 76,9 & 17,3 & 5,8 & 81,2 & 16,6 & 2,1 & 85,2 & 14,3 & 0,5 \\
\hline Setembro & 76,0 & 11,5 & 12,5 & 82,3 & 9,7 & 8,0 & 86,5 & 7,9 & 5,6 & 88,9 & 8,1 & 3,0 \\
\hline Outubro & 84,2 & 7,1 & 8,6 & 88,6 & 5,6 & 5,8 & 90,3 & 5,8 & 3,8 & 92,0 & 5,4 & 2,6 \\
\hline Novembro & 85,8 & 9,0 & 5,2 & 90,3 & 6,6 & 3,1 & 93,4 & 4,7 & 2,0 & 95,7 & 3,0 & 1,3 \\
\hline Dezembro & 89,2 & 5,3 & 5,5 & 92,4 & 4,1 & 3,5 & 94,6 & 3,8 & 1,6 & 96,7 & 2,8 & 0,5 \\
\hline
\end{tabular}


Tabela 4. Área agrícola (\%) da cana-de-açúcar cultivada na região Nordeste do Brasil com alto risco (AR), médio risco (MR) e baixo risco climático (BR) com redução de $25 \%$ na precipitação pluvial e sem aumento na temperatura do ar e aumentos de $1,5,3$ e $5{ }^{\circ} \mathrm{C}$

\begin{tabular}{|c|c|c|c|c|c|c|c|c|c|c|c|c|}
\hline \multirow{2}{*}{$\begin{array}{l}\text { Mês de } \\
\text { semeadura }\end{array}$} & AR & MR & BR & AR & MR & BR & AR & MR & BR & AR & MR & BR \\
\hline & \multicolumn{3}{|c|}{ Sem aumento } & \multicolumn{3}{|c|}{ Aumento de $1,5^{\circ} \mathrm{C}$} & \multicolumn{3}{|c|}{ Aumento de $3^{\circ} \mathrm{C}$} & \multicolumn{3}{|c|}{ Aumento de $5^{\circ} \mathrm{C}$} \\
\hline Fevereiro & 98,0 & 1,5 & 0,5 & 98,8 & 1,1 & 0,1 & 99,7 & 0,3 & 0,0 & 99,9 & 0,1 & 0,0 \\
\hline Abril & 99,9 & 0,1 & 0,0 & 100,0 & 0,0 & 0,0 & 100,0 & 0,0 & 0,0 & 100,0 & 0,0 & 0,0 \\
\hline Maio & 100,0 & 0,0 & 0,0 & 100,0 & 0,0 & 0,0 & 100,0 & 0,0 & 0,0 & 100,0 & 0,0 & 0,0 \\
\hline Junho & 99,7 & 0,3 & 0,0 & 100,0 & 0,0 & 0,0 & 100,0 & 0,0 & 0,0 & 100,0 & 0,0 & 0,0 \\
\hline Julho & 93,5 & 5,9 & 0,6 & 96,8 & 3,0 & 0,1 & 98,9 & 1,1 & 0,0 & 99,5 & 0,5 & 0,0 \\
\hline Outubro & 89,9 & 4,7 & 5,5 & 92,4 & 5,1 & 2,5 & 94,5 & 3,9 & 1,7 & 95,9 & 3,1 & 1,0 \\
\hline Novembro & 92,9 & 4,8 & 2,3 & 95,9 & 2,9 & 1,2 & 97,2 & 2,1 & 0,7 & 98,1 & 1,7 & 0,2 \\
\hline Dezembro & 94,6 & 3,3 & 2,1 & 96,2 & 3,1 & 0,7 & 97,9 & 2,0 & 0,1 & 99,3 & 0,6 & 0,0 \\
\hline
\end{tabular}

ao cultivo do algodoeiro está diretamente relacionada com a maior oferta hídrica dos cenários de aumento na precipitação pluvial. Considerando a permanência dos cenários de aumento na temperatura do ar, agora de $3{ }^{\circ} \mathrm{C}$, as reduções nas áreas com baixo e médio riscos climáticos ao cultivo da cana-de-açúcar são bastante significativas. Portanto, os segundos decêndios dos meses compreendidos entre fevereiro e agosto não apresentam áreas com baixo risco, sendo o período compreendido entre março e junho o mais sensível à variabilidade da precipitação, que afeta significativamente a disponibilidade das áreas agrícolas favoráveis ao cultivo da cana-de-açúcar exibindo, assim, $100 \%$ de áreas inaptas em todo o NEB. A redução das áreas agricultáveis é bem mais evidenciada com o aumento na temperatura de $5{ }^{\circ} \mathrm{C}$, sobretudo nas áreas com baixo risco climático, que cedem espaço para o aumento das áreas com alto risco, apresentando $83,3 \%$ de todo o período simulado, sem nenhuma área apta em todo o NEB. Campos et al. (2010) verificaram que, diante dos cenários de aumento na temperatura do ar, ocorre um aumento das áreas agrícolas com alto e médio riscos climáticos e, em contrapartida, uma redução nas áreas com baixo risco para todos os períodos simulados, resultados que indicaram que a atividade agrícola na região do NEB é bastante dependente da variação da temperatura do ar.

A Tabela 5 sumariza a variação percentual das áreas agrícolas da cana-de-açúcar cultivada na região Nordeste do Brasil com alto risco, médio risco e baixo risco climático, com um aumento de $25 \%$ na precipitação pluvial sem aumento na temperatura do ar e aumentos de $1,5,3$ e $5{ }^{\circ} \mathrm{C}$. Quando a variabilidade climática está associada à maior oferta hídrica, os cenários são um pouco mais animadores visto que para o cenário sem aumento na temperatura, nenhum dos períodos simulados apresenta áreas totalmente inaptas ao cultivo da cana-de-açúcar no NEB. O segundo decêndio do mês de maio é o que exibe a maior área com alto risco climático, com $98,5 \%$ que equivale a $1.506 .893,57 \mathrm{~km}^{2}$. Entretanto, as áreas agrícolas com baixo e médio risco climático, consideradas potencialmente favoráveis, apresentam um crescimento considerável na região em estudo enquanto o período compreendido entre os meses de julho, agosto, setembro e novembro são os mais otimistas de vez que as áreas agrícolas com baixo risco climático apresentam a maior área para este cenário, de $264.307,76 \mathrm{~km}^{2}$, sendo ainda maior que a área com médio risco, que exibe uma área de 224.100,64 $\mathrm{km}^{2}$ para o mesmo período.

Diante do cenário de aumento na temperatura de $1,5^{\circ} \mathrm{C}$ é possível observar que as áreas com aptidões agrícolas são sensivelmente reduzidas; contudo, o mês de agosto apresenta um acréscimo das áreas com médio risco climático em relação ao cenário anterior de $1,2 \%$, representando um aumento de $224.100,64 \mathrm{~km}^{2}$ para $243.173,26 \mathrm{~km}^{2}$ porém os segundos decêndios dos meses de abril e maio se exibem sem nenhuma área apta.

Investigando o cenário de aumento na temperatura de $3{ }^{\circ} \mathrm{C}$, as áreas agrícolas com baixo risco climático apresentam um período de três meses (abril a junho) sem nenhuma área apta ao

Tabela 5. Área agrícola (\%) da cana-de-açúcar cultivada na região Nordeste do Brasil com alto risco (AR), médio risco (MR) e baixo risco climático (BR) com aumento de $25 \%$ na precipitação pluvial e sem aumento na temperatura do ar e aumentos de $1,5,3$ e $5{ }^{\circ} \mathrm{C}$

\begin{tabular}{|c|c|c|c|c|c|c|c|c|c|c|c|c|}
\hline \multirow{2}{*}{$\begin{array}{c}\text { Mês de } \\
\text { semeadura }\end{array}$} & AR & $\overline{M R}$ & BR & $\overline{A R}$ & MR & BR & $\mathbf{A R}$ & MR & BR & $\overline{\mathbf{A R}}$ & MR & BR \\
\hline & \multicolumn{3}{|c|}{ Sem aumento } & \multicolumn{3}{|c|}{ Aumento de $1,5^{\circ} \mathrm{C}$} & \multicolumn{3}{|c|}{ Aumento de $3^{\circ} \mathrm{C}$} & \multicolumn{3}{|c|}{ Aumento de $5^{\circ} \mathrm{C}$} \\
\hline Janeiro & 90,9 & 4,6 & 4,5 & 94,0 & 2,7 & 3,3 & 95,7 & 2,5 & 1,7 & 97,5 & 2,1 & 0,4 \\
\hline Fevereiro & 93,5 & 3,6 & 2,9 & 95,6 & 2,8 & 1,6 & 97,3 & 2,2 & 0,5 & 99,0 & 1,0 & 0,1 \\
\hline Março & 95,6 & 3,4 & 1,1 & 97,6 & 2,1 & 0,3 & 98,9 & 1,0 & 0,1 & 99,8 & 0,2 & 0,0 \\
\hline Abril & 97,6 & 2,1 & 0,3 & 99,0 & 0,9 & 0,0 & 99,7 & 0,3 & 0,0 & 100,0 & 0,0 & 0,0 \\
\hline Maio & 98,5 & 1,5 & 0,0 & 99,7 & 0,3 & 0,0 & 100,0 & 0,0 & 0,0 & 100,0 & 0,0 & 0,0 \\
\hline Junho & 93,2 & 6,3 & 0,5 & 96,3 & 3,6 & 0,1 & 97,8 & 2,2 & 0,0 & 98,9 & 1,1 & 0,0 \\
\hline Julho & 75,3 & 17,0 & 7,7 & 81,8 & 14,3 & 3,8 & 86,8 & 11,2 & 2,0 & 92,4 & 6,8 & 0,7 \\
\hline Agosto & 68,1 & 14,7 & 17,3 & 73,9 & 15,9 & 10,2 & 78,3 & 17,4 & 4,3 & 81,6 & 16,7 & 1,7 \\
\hline Setembro & 73,2 & 12,7 & 14,1 & 79,4 & 11,0 & 9,6 & 84,0 & 9,1 & 6,9 & 86,9 & 8,3 & 4,8 \\
\hline Outubro & 81,5 & 9,0 & 9,5 & 87,3 & 5,8 & 6,9 & 89,2 & 6,2 & 4,6 & 90,9 & 5,7 & 3,4 \\
\hline Novembro & 83,8 & 9,6 & 6,6 & 88,1 & 8,0 & 3,9 & 91,6 & 5,8 & 2,6 & 94,3 & 3,9 & 1,8 \\
\hline Dezembro & 87,7 & 5,8 & 6,5 & 90,7 & 4,6 & 4,6 & 93,4 & 3,9 & 2,7 & 95,6 & 3,5 & 0,9 \\
\hline
\end{tabular}


cultivo da cana-de-açúcar enquanto o segundo decêndio do mês de maio é considerado o mais sensível para todos os cenários estudados, haja vista que as áreas com alto risco climático neste período, chegam facilmente a $100 \%$. Resultados semelhantes foram encontrados por Silva et al. (2012) no estudo sobre o cultivo do algodão herbáceo cultivado em sistema de sequeiro no Nordeste do Brasil, no cenário de mudança climática. Esses autores verificaram que o aumento da temperatura média do ar acima de $1,5^{\circ} \mathrm{C}$ se torna fator limitante ao cultivo do algodoeiro na região, mesmo diante do aumento da oferta hídrica. Apesar disto e embora persistindo os aumentos na temperatura do ar, o período simulado para o mês de agosto continua apresentando um crescimento das áreas com médio risco climático exibindo, para este cenário, $17,4 \%$ que corresponde a $265.975,19 \mathrm{~km}^{2}$.

$\mathrm{O}$ aumento na temperatura do ar de $5{ }^{\circ} \mathrm{C}$ provoca, neste mesmo período, uma pequena redução de $0,7 \%$ apresentando, assim, uma área com médio risco de 255.038,03 $\mathrm{km}^{2}$. Devido ao aumento na temperatura as áreas com baixo risco são severamente reduzidas quando comparadas com os cenários sem aumento na temperatura, apresentando quatro períodos (março a junho) sem áreas aptas ao cultivo; evidentemente, os períodos das áreas desfavoráveis crescem em relação ao cenário anterior de 1 para 2 meses com $100 \%$ de áreas inaptas. Analisando o impacto do aquecimento global sobre o cultivo de feijão caupi no Nordeste do Brasil, Silva et al. (2010) afirmam que o aumento na temperatura do ar afeta a disponibilidade de áreas agrícolas favoráveis e em particular a produtividade do feijão caupi no Nordeste do Brasil.

Nos cenários de redução de $40 \%$ na precipitação pluvial, sem aumento na temperatura do ar e aumentos de 1,$5 ; 3$ e 5 ${ }^{\circ} \mathrm{C}$, a oferta hídrica é reduzida drasticamente fazendo com que os riscos climáticos aumentem significativamente (Tabela 6). Desta maneira, as áreas agrícolas consideradas potencialmente favoráveis ao cultivo da cana-de-açúcar, sofrem um processo de redução bastante acentuado em todos os períodos simulados neste cenário. Mesmo sem aumento na temperatura, devido ao grande estresse hídrico, os segundos decêndios dos meses de abril a junho apresentam áreas com $100 \%$ de inaptidão agrícola ao cultivo da cana-de-açúcar; apenas os meses de agosto e setembro exibem uma área com médio risco climático considerável com 8,7 e 7,6\% da área total do NEB.

Considerando os aumentos na temperatura, os cenários associados ao decréscimo na precipitação pluvial tendem a aumentar as áreas com alto risco climático proporcionalmente; com o aumento na temperatura de $1,5^{\circ} \mathrm{C}$ as áreas agricultáveis com baixo risco climático são severamente reduzidas, com $58,3 \%$ dos períodos simulados sem nenhuma área apta ao cultivo da cana. As áreas consideradas desfavoráveis também apresentam um crescimento, com quatro períodos exibindo $100 \%$ de áreas inaptas ao cultivo da cana-de-açúcar. Por outro lado, o mês de setembro ainda apresenta $6,3 \%$ das áreas com médio risco climático representando $95.844,72 \mathrm{~km}^{2}$ de toda a área do NEB.

Analisando os resultados obtidos sob o cenário de aumento na temperatura de $3{ }^{\circ} \mathrm{C}$, verifica-se que, apesar de não apresentar nenhum período com áreas totalmente inaptas, ocorrem áreas agrícolas favoráveis com uma pequena variação de 0 a $0,9 \%$ para ás áreas com baixo risco climático e de 0,1 a 4,2\% para as áreas com médio risco. A deficiência hídrica afeta a produtividade da cana a qual, quando não satisfatória, pode acarretar baixo desenvolvimento das raízes e baixo perfilhamento resultando, portanto, num baixo aproveitamento da água e nutrientes disponíveis. A precipitação pluvial nas regiões brasileiras que produzem cana-de-açúcar é muito variável e má distribuída, sendo este o principal fator limitante da produtividade e, consequentemente, dos rendimentos agroindustriais desta cultura. Assim, algumas áreas se tornarão desfavoráveis em função do aumento no grau de risco climático associado ao cultivo da cana-de-açúcar na região.

Para o cenário de aumento na temperatura de $5{ }^{\circ} \mathrm{C}$ a época de cultivo da cana-de-açúcar é severamente reduzida fazendo com que a atividade agrícola tenha um risco maior associado ao clima. Neste cenário $66,6 \%$ dos períodos estudados apresentam áreas com alto risco climático com $100 \%$ de inaptidão agrícola ao cultivo da cana-de-açúcar. Segundo Silva et al. (2012) os riscos climáticos aumentam substancialmente quando o aumento da temperatura do ar está associado ao decréscimo de precipitação pluvial. Quanto maior a anomalia negativa de chuva menos apta se tornará a região.

Nos cenários de aumento na precipitação e mesmo com o acréscimo na temperatura do ar, verificam-se condições menos pessimistas em função, evidentemente, de uma oferta hídrica maior (Tabela 7). O cenário sem aumento na temperatura possibilita o aumento das áreas com baixo e médio risco climático, principalmente para o aumento de $40 \%$ na precipitação enquanto os segundos decêndios dos meses de

Tabela 6. Área agrícola (\%) da cana-de-açúcar cultivada na região Nordeste do Brasil com alto risco (AR), médio risco (MR) e baixo risco climático (BR) com redução de $40 \%$ na precipitação pluvial e sem aumento na temperatura do ar e aumentos de $1,5,3$ e $5{ }^{\circ} \mathrm{C}$

\begin{tabular}{|c|c|c|c|c|c|c|c|c|c|c|c|c|}
\hline \multirow{2}{*}{$\begin{array}{c}\text { Mês de } \\
\text { semeadura }\end{array}$} & $\overline{A R}$ & MR & BR & AR & MR & BR & $\mathbf{A R}$ & MR & BR & $\overline{A R}$ & MR & BR \\
\hline & \multicolumn{3}{|c|}{ Sem aumento } & \multicolumn{3}{|c|}{ Aumento de $1,5^{\circ} \mathrm{C}$} & \multicolumn{3}{|c|}{ Aumento de $3^{\circ} \mathrm{C}$} & \multicolumn{3}{|c|}{ Aumento de $5^{\circ} \mathrm{C}$} \\
\hline Janeiro & 98,0 & 1,3 & 0,7 & 98,8 & 1,0 & 0,1 & 98,0 & 1,1 & 0,9 & 100,0 & 0,0 & 0,0 \\
\hline Fevereiro & 99,0 & 0,9 & 0,1 & 99,6 & 0,3 & 0,0 & 98,3 & 1,4 & 0,4 & 100,0 & 0,0 & 0,0 \\
\hline Março & 99,8 & 0,2 & 0,0 & 100,0 & 0,0 & 0,0 & 98,6 & 1,2 & 0,1 & 100,0 & 0,0 & 0,0 \\
\hline Abril & 100,0 & 0,0 & 0,0 & 100,0 & 0,0 & 0,0 & 99,4 & 0,6 & 0,0 & 100,0 & 0,0 & 0,0 \\
\hline Maio & 100,0 & 0,0 & 0,0 & 100,0 & 0,0 & 0,0 & 99,9 & 0,1 & 0,0 & 100,0 & 0,0 & 0,0 \\
\hline Junho & 100,0 & 0,0 & 0,0 & 100,0 & 0,0 & 0,0 & 99,7 & 0,3 & 0,0 & 100,0 & 0,0 & 0,0 \\
\hline Julho & 98,1 & 1,9 & 0,1 & 99,6 & 0,4 & 0,0 & 98,5 & 1,3 & 0,2 & 100,0 & 0,0 & 0,0 \\
\hline Agosto & 91,2 & 8,7 & 0,0 & 96,6 & 3,4 & 0,0 & 98,5 & 1,3 & 0,2 & 100,0 & 0,0 & 0,0 \\
\hline Setembro & 89,8 & 7,6 & 2,7 & 93,5 & 6,3 & 0,2 & 95,8 & 4,2 & 0,0 & 98,7 & 1,3 & 0,0 \\
\hline Outubro & 92,0 & 5,3 & 2,7 & 94,8 & 3,8 & 1,4 & 96,7 & 2,9 & 0,5 & 98,2 & 1,8 & 0,0 \\
\hline Novembro & 95,7 & 2,9 & 1,3 & 97,6 & 2,1 & 0,3 & 96,3 & 3,4 & 0,3 & 99,3 & 0,7 & 0,0 \\
\hline Dezembro & 96,6 & 2,6 & 0,8 & 98,5 & 1,4 & 0,1 & 97,3 & 1,9 & 0,8 & 99,9 & 0,1 & 0,0 \\
\hline
\end{tabular}


Tabela 7. Área agrícola (\%) da cana-de-açúcar cultivada na região Nordeste do Brasil com alto risco (AR), médio risco (MR) e baixo risco climático (BR) com aumento de $40 \%$ na precipitação pluvial e sem aumento na temperatura do ar e aumentos de $1,5,3$ e $5{ }^{\circ} \mathrm{C}$

\begin{tabular}{|c|c|c|c|c|c|c|c|c|c|c|c|c|}
\hline $\begin{array}{c}\text { Mês de } \\
\text { semeadura }\end{array}$ & AR & MR & BR & AR & MR & BR & AR & MR & BR & AR & MR & BR \\
\hline Fevereiro & 92,3 & 4,0 & 3,8 & 94,5 & 3,2 & 2,4 & 96,5 & 2,6 & 0,9 & 98,3 & 1,5 & 0,1 \\
\hline Maio & 97,6 & 2,3 & 0,1 & 99,2 & 0,8 & 0,0 & 99,9 & 0,1 & 0,0 & 100,0 & 0,0 & 0,0 \\
\hline Junho & 90,6 & 8,4 & 1,0 & 94,8 & 4,9 & 0,2 & 96,6 & 3,3 & 0,1 & 98,2 & 1,8 & 0,0 \\
\hline Julho & 72,7 & 17,8 & 9,5 & 77,9 & 17,2 & 5,0 & 83,2 & 13,7 & 3,1 & 88,7 & 10,0 & 1,3 \\
\hline Outubro & 78,1 & 11,7 & 10,2 & 85,6 & 6,6 & 7,8 & 88,4 & 6,4 & 5,2 & 89,9 & 6,1 & 4,0 \\
\hline Novembro & 81,9 & 9,8 & 8,3 & 86,2 & 8,8 & 5,0 & 90,0 & 6,8 & 3,2 & 92,8 & 4,9 & 2,3 \\
\hline Dezembro & 86,0 & 5,9 & 8,1 & 88,8 & 5,7 & 5,5 & 92,2 & 4,3 & 3,5 & 94,5 & 3,8 & 1,7 \\
\hline
\end{tabular}

julho, agosto, setembro e outubro, exibem ótimo crescimento das áreas consideradas potencialmente favoráveis ao cultivo da cana-de-açúcar em relação aos cenários anteriores indicando a maior área apta no mês de agosto, com $295.732,58 \mathrm{~km}^{2}$. As áreas com alto risco climático sofreram, consequentemente, uma diminuição considerável, apresentando a maior área inapta de $97,6 \%$ para o segundo decêndio do mês de maio que equivale a $1.493 .499,86 \mathrm{~km}^{2}$ da área total do NEB. Para o cenário de aumento na temperatura de $1,5^{\circ} \mathrm{C}$, apenas o mês de maio exibe áreas com baixo risco climático nulo e um pequeno percentual de áreas com médio risco de $0,8 \%$. O período compreendido entre os meses de julho e setembro apresenta, praticamente, uma manutenção das áreas com médio risco climático tendo uma variação de 12,2 a 17,2\%. Novamente o período simulado para o mês de agosto se apresenta como o mês mais favorável ao cultivo da cana-de-açúcar na região Nordeste do Brasil com uma área apta de $206.811,55 \mathrm{~km}^{2}$.

Analisando o cenário de aumento na temperatura de $3{ }^{\circ} \mathrm{C}$, as áreas com alto risco climático surgem com mais significância anulando as áreas aptas no período de abril e maio; contudo, as áreas com médio risco garantem um pequeno percentual que varia de 0,6 e $0,1 \%$. Por outro lado, o mês de agosto exibe um crescimento das áreas com médio risco em relação ao cenário anterior de 15,4 para $17,6 \%$, representando um aumento de $33.161,03 \mathrm{~km}^{2}$. Portanto, os aumentos abruptos na temperatura também podem acarretar diminuição de áreas de médio e baixo riscos climáticos pois esta variável ambiental interfere no crescimento e no desenvolvimento das culturas, em razão de afetar significativamente a fenologia, a expansão foliar, a alongação dos internós, a produção de biomassa e a partição de assimilados em diferentes partes da planta; todavia, o cenário com aumento na temperatura de $5^{\circ} \mathrm{C}$ evidencia esta diminuição.

As áreas com baixo risco climático nula ocorrem em quatro períodos simulados (março a junho) diminuindo também sensivelmente as áreas com médio risco; apenas o mês de agosto mantém o mesmo percentual. Este cenário com forte aumento na precipitação pode ser comparado ao fenômeno La Niña com alta intensidade. Assim, percebe-se um único período, o mês de maio, apresentando áreas com alto risco climático com $100 \%$ de área inapta ao cultivo da cana-de-açúcar em toda a região do NEB. Para a região semiárida do Brasil e devido à grande irregularidade do seu regime pluviométrico, a conciliação dos períodos chuvosos e as melhores épocas de plantio são fatores determinantes para o sucesso da exploração agrícola de uma cultura (Campos et al., 2010). Por outro lado, Silva et al. (2005) constataram que a irregularidade do período chuvoso na região Nordeste do Brasil restringe a escolha de qualquer cultura a ser implantada, sobretudo na maior parte do semiárido, justamente onde o déficit hídrico é ainda maior.

\section{Conclusões}

1. As alterações climáticas provocadas pelo aumento na temperatura do ar e redução na precipitação pluvial provocam reduções significativas nas áreas agrícolas com baixo risco climático para a cultura da cana-de-açúcar cultivada na região Nordeste do Brasil.

2. As reduções na precipitação pluvial sem aumento na temperatura produzem um aumento significativo nas áreas com baixo e médio risco climático quando comparadas com aquelas submetidas aos incrementos de temperatura de $1,0.1,5$ e $5{ }^{\circ} \mathrm{C}$.

3. O período simulado mais sensível ao cultivo da canade-açúcar compreende os meses de março a maio enquanto os meses de julho a setembro é o período mais apropriado para o cultivo da cana-de-açúcar em sistema de sequeiro na região Nordeste do Brasil.

\section{Literatura Citada}

Almeida, A. C. S.; Souza, J. L.; Teodoro, I.; Barbosa, G. V. S.; Moura Filho, G.; Ferreira Júnior, R. A. Desenvolvimento vegetativo e produção de variedades de cana-de-açúcar em relação à disponibilidade hídrica e unidades térmicas. Ciência Agrotecnica, v.32, p.1441-1448, 2008.

Amaral, J. A. B.; Silva, M. T. Zoneamento Agrícola do Algodão Herbáceo no Nordeste Brasileiro Safra 2007/2008 - Estado do Ceará. Campina Grande: Embrapa CNPA, 2007. 8p. Comunicado Técnico 321

Baron, C.; Clopes, A.; Perez, P.; Muller, B.; Maraux, F. Manuels d'utilisation de: SARRAMET 45p SARRABIL 35p et SARRAZON 29p. Montpellier: CIRAD. 1996.

Baron, C.; Sultan, B.; Balme, M.; Sarr, B.; Traoré, S.; Lebel, T.; Janicot, S.; Dingkuhn, M. From GCM grid cell to agricultural plot: Scale issues affecting modeling of climate impact. Philosophical Transactions of Royal Society B, v.360, p.2095-2108, 2005. 
Campos, J. H. B. C.; Silva, M. T.; Silva, V. de P. R. da. Impacto do aquecimento global no cultivo do feijão-caupi, no Estado da Paraíba. Revista Brasileira de Engenharia Agrícola e Ambiental, v.14, p.396-404, 2010.

Chavas, D. R.; Izaurralde, R. C.; Thomson, A. M.; Gao, $\mathrm{X}$. Long-term climate change impacts on agricultural productivity in eastern China. Agricultural and Forest Meteorology, v.149, p.1118-1128, 2009.

Cavalcanti, E. P.; Silva, V. de P. R. da; Sousa, F. A. S. Programa computacional para a estimativa da temperatura do ar para a região Nordeste do Brasil. Revista Brasileira de Engenharia Agrícola e Ambiental, v.10, p.140-147, 2006.

Doorenbos, J.; Pruitt, W. Guidelines for predicting crop water requirements. Rome: FAO, 1977.144p. Irrigation and Drainage Paper, 24

EPE - Empresa de Pesquisa Energética. Balanço Energético Nacional 2008: Ano base 2007-Rio de Janeiro: EPE, 2008. $244 p$.

Farias. C. H. de A.; Fernandes, P. D.; Azevedo, H. M.; Dantas Neto, J. Índices de crescimento da cana-deaçúcar irrigada e de sequeiro no estado da Paraíba. Revista Brasileira de Engenharia Agrícola e Ambiental, v.12, p.356-362, 2008.

Harmsen, E. W.; Miller, N. L.; Schlegel, N. J.; Gonzalez, J. E. Seasonal climate change impacts on evapotranspiration, precipitation deficit and crop yield in Puerto Rico. Agricultural Water Management, v.96, p.1085-1095, 2009.

IPCC - Intergovernmental Panel on Climate Change. Climate Change 2001: Impacts, adaptation and vulnerability. Working Group II. TAR: Summary for Policymakers. http:// www.meto.gov.uk/sec5/CR_div/ipce/wg1/WG1-SPM.pdf. 21 Jul. 2010.

IPCC - Intergovernmental Panel on Climate Change. Climate Change 2007: The physical science basis. Summary for policy makers contribution of Working Group I to the Fourth Assessment Report of the Intergovernmental Panel on Climate Change. http://ipcc-wg1.ucar.edu/wg1/wg1report.html. 21 Jul. 2010.
Kouressy, M.; Dingkuhn, M.; Vaksmann, M.; Heinemann, A. B. Adaptation to diverse semi-arid environments of sorghum genotypes having different plant type and sensitivity to photoperiod. Agricultural and Forest Meteorology, v.148, p.357-371, 2008.

Lohmann, S.; Schillings, C.; Mayer, B.; Meyer, R. Long-term variability of solar direct and global radiation derived from ISCCP data and comparison with reanalysis data. Solar Energy, v.80, p.1390-1401, 2006.

Mishra, A.; Hansen, J. W.; Dingkuhn, M.; Baron, C.; Traoré, S. B.; Ndiaye, O.; Ward, M. N. Sorghum yield prediction from seasonal rainfall forecasts in Burkina FAO. Agricultural and Forest Meteorology, v.148, p.1798-1814, 2008.

Oliveira, S. D. de; Silva, V. de P. R. da; Santos, Carlos A. C. dos; Silva, M. T.; Sousa, E. P. de. Os impactos das alterações climáticas na cana-de-açúcar cultivada em sistema de sequeiro na região nordeste do Brasil. Revista Brasileira de Geografia Física, v.5, p.170-184, 2012.

Silva, M. T.; Silva, V. de P. R. da; Azevedo, P. V. de. O cultivo do algodão herbáceo no sistema de sequeiro no nordeste do Brasil, no cenário de mudanças climática. Revista Brasileira de Engenharia Agrícola e Ambiental, v.16, p.80-91, 2012.

Silva, V. de P. R. da. On climate variability in Northeast of Brazil. Journal of Arid Environments, v.58, p.575-596, 2004.

Silva, V. de P. R. da; Belo Filho, A. F.; Silva, B. B. da; Campos, J. H. B. C. Desenvolvimento de um sistema de estimativa da evapotranspiração de referência. Revista Brasileira de Engenharia Agrícola e Ambiental, v.9, p.547-553, 2005.

Silva, V. de P. R. da; Campos, J. H. B. C.; Silva, M. T.; Azevedo, P. V. Impact of global warming on cowpea bean cultivation in northeastern Brazil. Agricultural Water Management, v.97, p.1760-1768, 2010.

Sultan, B.; Baron, C.; Dingkuhn, M.; Sarr, B.; Janicot, S. Agricultural impacts of large-scale variability of the West African monsoon. Agricultural and Forest Meteorology, v.128, p.93-110, 2005.

Thornton, P. K.; Jones, P. G.; Alagarswamy, G.; Andresen, J. Spatial variation of crop yield response to climate change in East Africa. Global Environmental Change, v.19, p.54-65, 2009. 